\title{
BRIEF
}

\section{A Pilot Study Assessing the Effect of a Transitions of Care Simulation on Student Empathy}

\author{
Elizabeth Ann Cook, PharmD, Jessica Wooster, PharmD, Frank Yu, PharmD
}

Robert J. Dole Veteran Affairs Medical Center, Wichita, Kansas

Corresponding Author: Elizabeth Ann Cook, Robert J. Dole Veteran Affairs Medical Center, 9211 W. Briarwood Ave., Wichita, KS 67212. Tel: 903-565-6531. Email: elizabethcookpharmd@gmail.com

Submitted December 30, 2020; accepted May 25, 2021; ePublished June 2021

Objective. To assess the effects of a transitions of care (TOC) simulation on the empathy of pharmacy students. Methods. Pharmacy students volunteered to complete a four-hour TOC simulation. Students were "discharged" from a simulation hospital by trained actors posing as health care providers and provided with a discharge packet, prescriptions, bus route and bus pass. Students navigated public transportation to obtain discharge medications at a community pharmacy and then returned "home" to debrief with study investigators. Demographics were analyzed using descriptive statistics. The Kiersma-Chen Empathy Scale (KCES) was administered pre- and post-simulation, along with open-ended questions.

Results. Median composite empathy scores of the KCES increased significantly from 92 to 98 following completion of the simulation. Statistically significant increases were seen in four of the fifteen singular questionnaire items whose themes largely involved taking patients' feelings into account with therapeutic decision making. These were in the items "I will not allow myself to be influenced by someone's feelings when determining the best treatment," "I have difficulty identifying with someone else's feelings," "It is necessary for a health care practitioner to be able to view the world from another person's perspective," and "A health care practitioner should not be influenced by someone's feelings when determining the best treatment."

Conclusion. Results of this pilot demonstrated a significant increase in overall empathy among student pharmacists. Replicating this experiment on a larger scale may provide further insight regarding the impact of simulations revolving around TOC on empathy of student pharmacists.

Keywords: simulation, transitions of care, empathy, pharmacy students, attitudes

\section{INTRODUCTION}

Transitions of care (TOC) is defined as the movement of patients between health care settings, providers, or different levels of care as conditions or care needs change. ${ }^{1}$ As health care in the United States has shifted from fee-forservice to quality-based care, an emphasis has been placed on reducing readmissions of patients discharged from the hospital to the outpatient setting. ${ }^{2,3}$ To reduce hospital readmissions, the National Transitions of Care Coalition has emphasized integrating various health care professions, including pharmacists, into the discharge coordination process. While use of an interprofessional care team has been shown to improve TOC related outcomes, coordinating care for persons with limited social support may still be challenging. ${ }^{1,3,4}$

Some patients transitioning from hospital to home assume sole responsibility of following through on discharge instructions, communicating with outpatient providers, and attending follow-up appointments for their health conditions, in addition to actively recovering from an acute illness. ${ }^{5,6}$ If patients lack the resources or understanding to accomplish these self-management strategies, continuity of care may collapse, which can potentially result in hospital readmission and emotional distress for the patient. ${ }^{7}$ That being said, health care professionals' ability to empathize with a patient's hardships has been strongly associated with improved outcomes in medical care in several disease states and care settings. ${ }^{8-10}$

Thus, empathy is a necessary component in the development of a functional patient-provider relationship. ${ }^{9,10}$ To instill empathy in students training in health care professions, exercises have been incorporated into the didactic and experiential curricula of several disciplines. Evidence has shown that experiential education and active learning exercises, such as simulations, are effective tools to instill empathy in students enrolled in nursing, physician, and pharmacy coursework. ${ }^{11-18}$ As TOC is an evolving topic in health care, few studies to date have been conducted to detail how this 
subject should be incorporated into health education curricula, or how students perceive the hardships of patients transitioning from one health care setting to another with limited resources or social support.

This study serves to describe the impact of a TOC simulation on student understanding of the complexity of care transitions, importance of the pharmacist's role in this process, and to measure if such a scenario can result in increased empathy towards underserved patients experiencing a hospital discharge. Additionally, this study serves as a pilot to help faculty determine if it would be worthwhile to devote the resources to replicate this simulation on a larger scale for empathy development.

\section{METHODS}

This exploratory study voluntarily recruited first- through fourth-year pharmacy students at The University of Texas at Tyler (UT Tyler), Ben and Maytee Fisch College of Pharmacy to complete a four-hour simulation in the life of a patient discharged from inpatient care following an acute illness. A secure email was sent out by an administrative assistant to all student pharmacists within the college for recruitment. All students participating voluntarily in the study were compensated for their time with a gift card valued at 20 USD and it was emphasized that participation would not factor into grades for any course. This study was granted exemption status by UT Tyler Institutional Review Board.

The timeline of the simulation study is outlined in Figure 1. During the Briefing Phase, two participants at a time were scheduled to present to the UT Tyler Nursing Simulation Laboratory, a facility that replicates the environment of an inpatient internal medicine unit. Participants were oriented to the patient whose role they would be assuming, a middleaged individual who had suffered their first myocardial infarction and must now pick-up their discharge medications from a community pharmacy. The patient was stated to have no close family members, personal mode of transportation, or sufficient means to call a cab/ride share service, requiring participants to navigate public transportation.

Participants were placed in separate hospital rooms to begin the Discharge Phase of the simulation. Trained actors, posing as a provider and a nurse, conducted scripted discharge education sessions that were timed to allow for equal attention among study subjects. The participants were then provided with a discharge packet containing their hospital records, several hardcopy prescriptions for new home medications, a bus schedule, a bus pass, the address of the pharmacy where they would fill their prescriptions, and directions to the nearest bus stop.

After receiving the above supplies, participants then entered the Attainment Phase, boarding public transit to travel to a community pharmacy where they would fill their prescriptions. At a designated community pharmacy, students provided their faux prescriptions to a pharmacist recruited by the study investigators, who would then fill the prescriptions and provide brief counseling for the medications. After leaving the community pharmacy, students would enter the Return Phase of the simulation, where they would utilize public transportation to return "home" to the University campus. Students then walked to the college of pharmacy to debrief with investigators. One investigator ensured that the entire simulation could be completed in approximately four hours by completing the simulation three separate times, by waiting at stations where participants interacted with actors, walking to key locations, and riding public transportation themselves to clock the total duration. Fluctuations in time to complete the simulation may have occurred among participants given delays in public transportation, lines at the pharmacy, or possible confusion with the bus route.

Students anonymously completed a pre-simulation survey containing demographic questions, information regarding previous experience in health care and hospital discharge processes (Table 1), as well as the Kiersma-Chen Empathy Scale (KCES). The KCES is a questionnaire with 15 items presented in a Likert-type format $(1=$ strongly disagree to $7=$ strongly agree) that has been validated for use in pharmacy and nursing students. Both affective qualities (the ability to relate to another individual's emotional and mental state), as well as cognitive qualities (the ability to view situations from another individual's perspective), are assessed with this questionnaire, with higher scores correlating with a higher level of empathy. ${ }^{19}$

Post-simulation, students repeated the KCES, as well as completed the 3-item Care Transition Measure (CTM-3), a survey validated for use in persons being discharged from emergency and/or inpatient care in the United States. Items in the CTM-3 address patients' perceptions of hospital staff's respect for their preferences and health care needs, their understanding of self-management strategies for their health conditions, as well as their understanding of medication indications at hospital discharge.$^{20}$ Additionally, participants were presented with two open-ended questions to reflect on their experiences during the simulation, particularly their thoughts on the use of public transportation, as what could possibly be done to improve patient transitions from hospital discharge to community pharmacies.

Data analysis was performed using IBM SPSS Statistics for Windows, Version 24.0. (IBM Corp., Armonk, N.Y., USA). Descriptive statistics were used to report student demographics such as gender, age, ethnicity, pharmacy year, prior use of public transportation, prior hospitalizations, and experience as a caregiver. The Wilcoxon signed-rank test was used to analyze changes in each KCES item before and after the simulation as well as changes in the composite score. The level of statistical significance was set at $\alpha=0.05$. A thematic analysis was used to report open-ended responses from the 
questions pertaining to the simulation experience. Two investigators developed an initial scheme to holistically code the results of the open-ended survey questions based on recurring, similarly meaning words and phrases identified in the responses. After inductively coding the data, the results were shared with the entire research team to reach consensus on the clarity and structure of the framework, as well as discuss subcodes within the themes identified. ${ }^{21}$

\section{RESULTS}

Of the 315 potential students who received the recruitment email, thirteen students participated in the simulation and completed both the pre-simulation and post-simulation survey materials. Demographic information of participants is listed in Table 1 Prior to the simulation, $46.2 \%$ of participants had never used public transportation, $61.2 \%$ had never been hospitalized for an acute illness, and while all participants had a family member or friend hospitalized for an acute illness, $76.9 \%$ had never served as a caregiver for those affected individuals.

Post-simulation, statistically significant increases in empathy were seen in items $4(p=.029), 11(p=.024), 14(p=$ $.005)$, and $15(p=.009)$ in the KCES (Table 2). Despite the majority of individual items not showing a change in empathy, there was a statistically significant increase in empathy in the overall composite score $(p=.003)$.

Regarding the results of the post-simulation CTM-3 survey, for the item pertaining to hospital staff taking patient preferences into account in developing care needs after leaving the hospital, 30\% of participants strongly agreed, $8 \%$ agreed, $38 \%$ disagreed, $8 \%$ strongly disagreed, and $15 \%$ could not recall whether this was achieved when they were "discharged." Results of the item on participants feeling that they understood the tasks they were responsible for in managing their health, $23 \%$ strongly agreed, $16 \%$ agreed, $23 \%$ disagreed and 38\% strongly disagreed with this statement. For the final item, results of the participants understanding the indications of their discharge medications prior to being discharged, $8 \%$ strongly agreed, $8 \%$ agreed, $38 \%$ disagreed, and $46 \%$ strongly disagreed with that statement. Several common themes were identified in the two open-ended reflection questions about their experience. Aside from the need for increased empathy, the most frequent response to improve the transition of patients from the hospital to community pharmacy was the need for improved communication between health care providers and the patient, followed by the need for communication amongst health care providers to convey changes in the patient's health status and medical treatment plan. Additionally, participants frequently noted the need for more thorough discharge education, particularly medication indication and administration instructions, assurance of patient understanding, and outcomes of possible medication nonadherence. Many students also provided ideas to overcome transportation issues including organizing transportation for discharged patients to their chosen pharmacy to pick up medications, providing a supply of medications at the time of discharge, or delivering new medications directly to the home of the patient to improve medication adherence and decrease stress.

When asked about their perceptions regarding use of public transportation after hospital discharge, most participants described the process as complex and time-consuming, detailing the confusing bus routes despite having a map, long waits at the bus stop, and the long rides to only travel a short distance. It was also described as an exhausting method of transportation as they had to take long walks to and from bus stops and desired destinations, which was noted as a barrier specifically in patients with mobility issues. All students remarked that the process was exhausting for them as overall healthy individuals and thus it would be especially tiring in patients after being acutely ill. Zero participants reported that they became lost or missed boarding times when navigating public transportation.

\section{DISCUSSION}

Hospital readmissions place a significant burden on patients and the health care system. ${ }^{1,2}$ It is important for health care professionals to be able to anticipate patient, provider, and health system-related barriers that may result in asynchronous care in order to reduce the likelihood of hospital readmission. ${ }^{1,4}$

The objective of this pilot study was to assess changes in pharmacy students' empathy towards patients undergoing transitions of care from a hospital to community setting. Our study results demonstrated an overall increase in student empathy from baseline, with statistically significant increases in empathy on four of the fifteen questionnaire items, three of which dealt with the affective domain.

Results of the thematic analysis suggest that the simulation provided participants with insight into hardships commonly encountered by those with limited financial resources and/or personal support systems. It also revealed that students identified the disconnect in communication between the health care provider and patient, as well as the mentally and physically taxing nature of navigating public transportation, as areas which may impact underserved patients the most when undergoing transitions of care. These observations appeared to support the statistically significant increases in the cognitive and affective empathy domains in the questionnaire. ${ }^{29}$

Although a variety of activities have been conducted to highlight difficulties that specific patient populations may experience, to our knowledge, this is the first study to investigate whether a TOC simulation will improve student 
pharmacists' empathy towards underserved patients. Incorporation of simulated activities in pharmacy school curricula has been shown to increase learners' ability to relate to the hardships that patients may undergo when managing acute and chronic health conditions. ${ }^{11,12,14}$ The results of this study, demonstrating an increase in student empathy following activity completion, are congruent with others examining changes in learner empathy towards patients in differing scenarios. ${ }^{11-18}$

However, certain limitations should be considered when drawing conclusions from this study. While student empathy appeared to increase following the simulation, most of the survey items did not demonstrate statistically significant changes. Results of the pre-simulation KCES illustrated that participating students had a relatively high baseline empathy score, which could possibly justify the lack of a significant increase in scores of certain survey items. This may have been influenced by the fact that this activity took place outside of existing coursework, allowing for participant self-selection, thus introducing the potential for volunteer bias. The purpose and theme of the study may have also attracted individuals who were more susceptible to the aim of the intervention compared to those students who did not volunteer. ${ }^{22}$

As volunteers were first- through fourth-year student pharmacists, exposure to TOC concepts was highly variable across the participant pool, which may limit generalizability of this study's impact to one specific cohort of learners. It could be posited that students who had previously been exposed to concepts of TOC in introductory or advanced pharmacy practice experience rotations were better able to empathize with patients due to their real-world experience on the subject matter. It is also unknown as to whether the changes in empathy instilled from this activity would be sustained long term throughout the remainder of the participants' education or post-graduation when they enter real-world practice.

Furthermore, the sample size of this pilot study was limited by available funds, personnel, equipment, scheduling, and space. While the small number of participants offered advantages, such as limiting logistical problems and cost, it may also have contributed to type II error, decreasing the researchers' ability to truly detect a difference in the participants KCES scores at the end of the simulation. Ultimately, this pilot study primarily served as a gauge of feasibility in implementing this intervention on a larger scale, which carries utility in and of itself. It is also worth mentioning that while pre- and post-simulation data offered a valuable perspective, solid conclusions regarding the utility of this activity cannot necessarily be gleaned without a comparison to those students who were educated about the hardships of TOC using traditional teaching methods in the college's existing curriculum.

\section{CONCLUSION}

These results of this hospital to community transitions of care simulation suggest that use of such scenarios to demonstrate potential barriers to facilitate self-care after discharge may improve empathy among student pharmacists. This study helps establish a need for future simulations examining student pharmacist's understanding of the complexity of care transitions, importance of the pharmacist's role in this process, and whether engagement in such activities can result in increased empathy towards underserved patients at hospital discharge.

\section{ACKNOWLEDGMENTS}

This research was supported by a seed grant from The University of Texas at Tyler, Ben and Maytee Fisch College of Pharmacy. We thank Erica Chase, CPhT for her service as an acting coach, as well as the actors who served during the course of the simulation. We also thank Nicholas Garner, PharmD for his service as the community pharmacist in the simulation.

\section{REFERENCES}

1. The National Transitions of Care Coalition. Improving transitions of care: the vision of the national transitions of care coalition. May 2008. Available at https://www.ntocc.org/s/PolicyPaper.pdf. Accessed April 10, 2021.

2. Medicare Payment Advisory Commission. Report to the Congress: Promoting Greater Efficiency in Medicare. June 2007. Chapter 5. Available at http://www.medpac.gov/documents/jun07_EntireReport.pdf. Accessed May 7, 2020.

3. Institute of Medicine (US) Committee on Identifying Priority Areas for Quality Improvement, Adams K, Corrigan JM, eds. Priority Areas for National Action: Transforming Health Care Quality. Washington (DC): National Academies Press (US); 2003. doi: 10.17226/10593

4. Readmissions reductions program. CMS. Updated August 2013. Available at www.cms.gov/Medicare/MedicareFee-for-Service-Payment/AcuteInpatientPPS/Readmissions-Reduction-Program.html. Accessed May 7, 2020

5. Rattray NA, Sico JJ, Cox LM, Russ AL, Matthias MS, Frankel RM. Crossing the Communication Chasm: Challenges and Opportunities in Transitions of Care from the Hospital to the Primary Care Clinic. Jt Comm J Qual Patient Saf. 2017;43(3):127-137. doi:10.1016/j.jcjq.2016.11.007 
6. Mansukhani RP, Bridgeman MB, Candelario D, Eckert LJ. Exploring Transitional Care: Evidence-Based Strategies for Improving Provider Communication and Reducing Readmissions. P T. 2015;40(10):690-694.

7. Christman NJ, McConnell EA, Pfeiffer C, Webster KK, Schmitt M, Ries J. Uncertainty, coping, and distress following myocardial infarction: transition from hospital to home. Res Nurs Health. 1988;11(2):71-82. doi:10.1002/nur.4770110203

8. Hojat M, Louis DZ, Markham FW, Wender R, Rabinowitz C, Gonnella JS. Physician Empathy and clinical outcomes for diabetic patients. Acad Med. 2011;86(3):359-364.

9. Hojat M. Empathy in patient care: antecedents, development, measurement, and outcomes. New York: Springer, 2007.

10. Reiss II. Empathy in medicine: a neurologic perspective. J Am Med Assoc. 2010;304(14);1604-1605.

11. Chen AMH, Kiersma ME, Yehle KS, Plake KS. Impact of an aging simulation game on pharmacy students' empathy for older adults. Am J Pharm Educ. 205;79(5):Article 65.

12. Chen JT, LaLopa J, Dang DK. Impact of patient empathy modeling on pharmacy students caring for the underserved. Am J Pharm Edu. 2008;72(2):Article 40.

13. Mennenga HA, Bassett S, Pasquariello L. Empathy development through case study and simulation. Nurse Educator. 2016;41(4):139-142.

14. Van Winkle LJ, Fjortoft N, Hojat M. Impact of a workshop about aging on the empathy scores of pharmacy and medical students. Am J Pharm Educ. 2013;76(1):9.

15. Lor KB, Truong JT, Ip EJ, Barnett MJ, A randomized prospective study on outcomes of an empathy intervention among second-year student pharmacists. Am J Pharm Educ. 2015;79(2):Article 9.

16. Fjortoft N, Van Winkle LJ, Hojat M. Measuring empathy in pharmacy students. Am J Pharm Educ. 2011;75(6):Article 109.

17. Hojat M, Gonnella JS, Mangione S, et al. Empathy in medical students as related to clinical competence, gender and academic performance. Med Educ. 2002;36(6):522-527.

18. Hojat M, Mangione S, Nasca TJ, Gonnella JS. Empathy scores in medical school and ratings of pharmacist empathic behavior 3 years later. J Soc Psychol. 2005;145(6):663-672.

19. Kiersma ME, Chen AM, Yehle KS, Plake KS. Validation of an empathy scale in pharmacy and nursing students. Am J Pharm Educ. 2013;77(5):94. doi:10.5688/ajpe77594

20. Parry C, Mahoney E, Chalmers SA, Coleman EA. Assessing the quality of transitional care: further applications of the care transitions measure. Med Care. 2008 Mar;46(3):317-22. doi: 10.1097/MLR.0b013e3181589bdc.

21. Saldana, J. The coding manual for qualitative researchers. $2^{\text {nd }}$ ed. Thousand Oaks, CA, SAGE Publications, 2013.

22. Brownell SE, Kloser MJ, Fukami T, Shavelson RJ. Context matters: volunteer bias, small sample size, and the value of comparison groups in the assessment of research-based undergraduate introductory biology lab courses. J Microbiol Biol Educ. 2013 Dec 2;14(2):176-82. doi: 10.1128/jmbe.v14i2.609.

23. Saldaña J. The coding manual for qualitative researchers. $2^{\text {nd }}$ ed. Thousand Oaks, CA: SAGE Publications, 2013 
Table 1 Demographics of Pharmacy Students Participating in a Transitions-of-Care Simulation

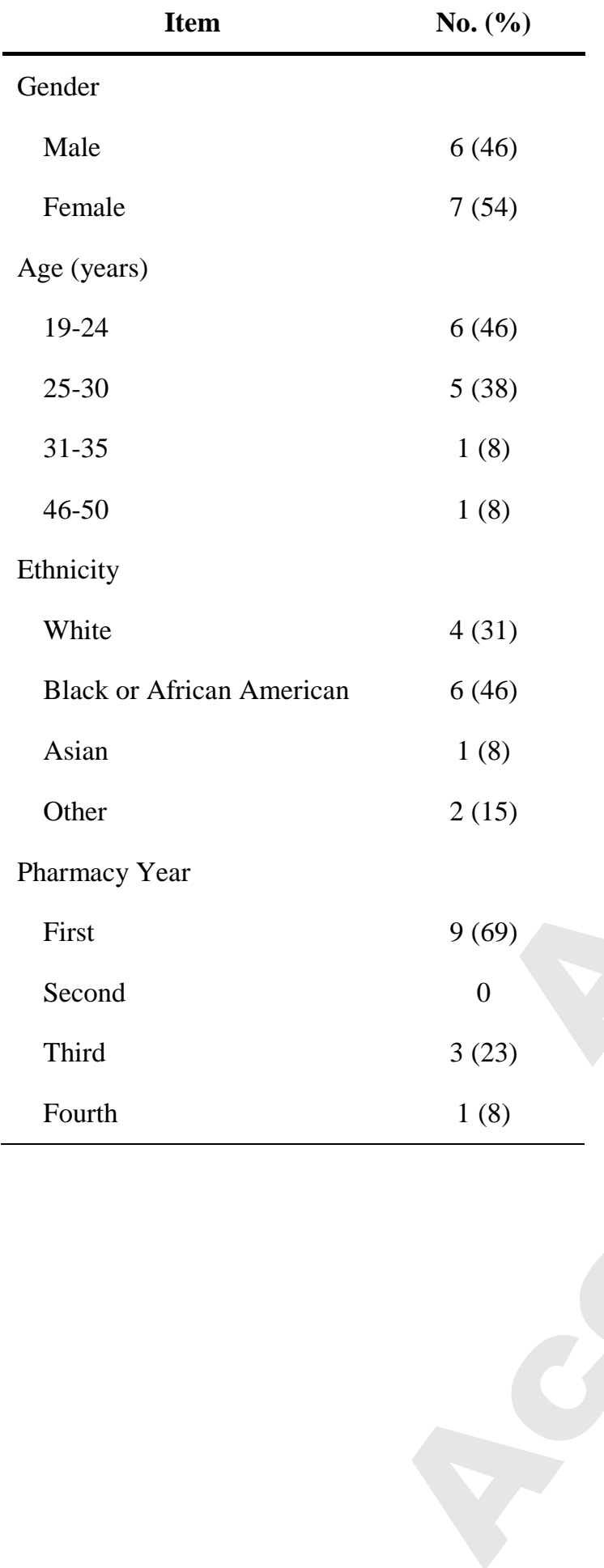


Table 2. Comparison of Pharmacy Students Kiersma-Chen Empathy Scale Scores Before and After Transition-of-Care Simulation Activity

\begin{tabular}{ccc} 
KCES Item & $\begin{array}{c}\text { Pre-Simulation } \\
\text { Median Score }(I Q R)^{a}\end{array}$ & $\begin{array}{c}\text { Post-Simulation } \\
\text { Median Score (IQR) }\end{array}$ \\
\hline
\end{tabular}

$7(0)$

$7(0)$

NS

1. It is necessary for a health care practitioner to be able to comprehend someone else's experiences.

$6(1)$

$6(1)$

NS someone's feelings.

$6(2)$

$7(1)$

NS experiences. able to express an understanding of someone's feelings.

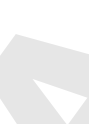

6. It is necessary for a health care practitioner to be able to value someone else's point of view.

7. I believe that caring is essential to building a strong relationship with patients.

8. I am able to view the world from another person's perspective.

9. Considering someone's feeling is not necessary to provide patient-centered care.

11. I have difficulty identifying with someone else's 
feelings.

12. To build a strong relationship with patients, it is essential for a health care practitioner to be caring.

13. It is necessary for a health care practitioner to be able to identify with someone else's feelings.

14. It is necessary for a health care practitioner to be able to view the world from another person's perspective.

15. A health care practitioner should not be influenced by someone's feelings when determining the best treatment.

Overall composite score

KCES $=$ Kiersma-Chen Empathy Scale; IQR=Interquartile Range

${ }^{a}$ Scale: 1-7 ( $1=$ strongly disagree, $2=$ disagree, $3=$ somewhat disagree, $4=$ neutral, $5=$ somewhat agree, $6=$ agree, $7=$ strongly agree $)$

${ }^{\mathrm{b}}$ Item inversely scored; lower post-simulation value reflects increase in empathy 


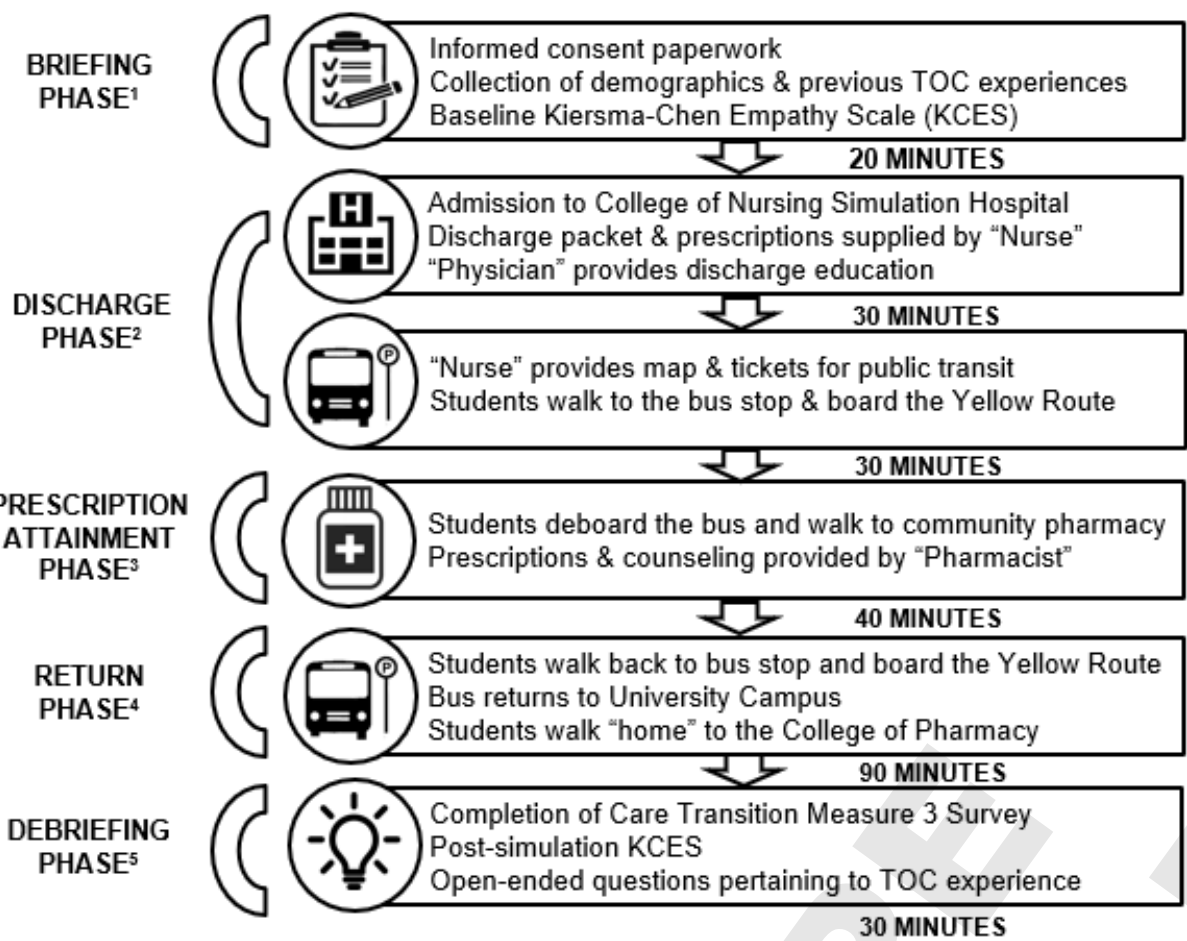

1. Student pharmacists completed informed consent paperwork, baseline demographics survey, as well as the Kiersma Chen Empathy Scale within 20 minutes prior to engaging in simulation. Students were then oriented to the patient whose role they would be assuming for the simulation.

2. Participants were placed in separate hospital rooms and underwent discharge counseling by trained actors posing as a physician and a nurse. Participants were provided with a discharge packet containing prescriptions for their discharge medications, a bus schedule, directions, and the address of the pharmacy where they would fill their prescriptions. They then walked to the bus stop to board public transportation.

3. Participants deboarded the bus and presented to a community pharmacy where a pharmacist participating in the simulation filled faux prescriptions and provided medication counseling.

4. Students walked back to the bus stop to travel to the University campus and deboarded the bus to walk "home" to the college of pharmacy

5. Students completed the Care Transition Measure 3, KCES, as well as open ended questions pertaining to their learning experiences from the simulation. Students were then dismissed from the simulation exercise. 